\title{
NANOSUSPENSION: A MODERN TECHNOLOGY USED IN DRUG DELIVERY SYSTEM
}

\author{
HRISHAV DAS PURKAYASTHA*, S. K. IMANUR HOSSIAN
}

Acharya and BM Reddy College of Pharmacy, Achit Nagar, Bengaluru 560090

Email: vardwaj08@gmail.com

Received: 15 Feb 2019, Revised and Accepted: 11 Apr 2019

\begin{abstract}
Nanosuspension consists of the pure poorly water-soluble drug without any matrix material suspended in dispersion. The formulation as nanosuspension is an attractive and promising alternative to solve these problems. Nanosuspension technology solved the problem of drugs which are poorly aqueous soluble and less bioavailability. Stability and bioavailability of the drugs can be improved by Nanosuspension technology. Nanosuspensions are promising candidates that can be used for enhancing the dissolution of poorly water-soluble drugs. Preparation of Nanosuspension is simple and applicable to all drugs which are aqueous insoluble. Nanosuspensions are prepared by using wet mill, high-pressure homogenizer, emulsion-solvent evaporation, melt emulsification method and supercritical fluid techniques. Nanosuspension can be prepared by using stabilizers, organic solvents and other additives such as buffers, salts, polyols, osmogent and cryoprotectant. Nanosuspensions can be delivered by oral, parenteral, pulmonary and ocular routes. Nanosuspensions can also be used for targeted drug delivery when incorporated in the ocular inserts and mucoadhesive hydrogels.
\end{abstract}

Keywords: Nanosuspension, Bioavailability, Emulsification, Homogenization, Stability

(C) 2019 The Authors. Published by Innovare Academic Sciences Pvt Ltd. This is an open-access article under the CC BY license (http://creativecommons.org/licenses/by/4.0/) DOI: http://dx.doi.org/10.22159/ijcpr.2019v11i3.34098

\section{INTRODUCTION [1-4]}

One of the main problems responsible for the low turnout in the development of new molecular entities drug formulations is poor solubility and poor permeability of the lead compounds. More than $40 \%$ of the new chemical entities being generated through drug discovery programmes are poorly water-soluble or lipophilic compounds. Formulating a poorly water solubledrug has always been a challenging problem confronted by the pharmaceutical scientist. A Pharmaceutical Nanosuspension is a biphasic liquid system in which insoluble solid drug particles are uniformly dispersed in an aqueous vehicle. The dosage form is colloidal in nature and usually stabilized by surfactant and polymers. The formulation of nano-sized particles can be implemented to all drug compounds belonging to the biopharmaceutical classification system (BCS) classes II and IV to increase their solubility. There are many conventional methods for increasing the solubility of poorly soluble drugs, which include micronization, solubilization using co-solvents, salt form, surfactant dispersions, precipitation technique, and oily solution. Other techniques are like liposomes, emulsions, microemulsion, solid dispersion and inclusion complexation using cyclodextrins. Nanosuspension technology can also be used for drugs which are insoluble in both water and organic solvents. Hydrophobic drugs such as Atorvastatin, Famotidine, Simvastatin, Revaprazan, Aceclofenac, are formulated as Nanosuspension.

For a long duration of time micronization of poorly soluble drugs by colloid mills or jet mills was preferred. The overall particle size distribution ranges from $0.1 \mu \mathrm{m}$ to approximately $25 \mu \mathrm{m}$, the only negligible amount being below $1 \mu \mathrm{m}$ in the nanometer range.

Nanosuspensions are colloidal dispersions of nanosized drug particles stabilized by surfactants. They can also be defined as a biphasic system consisting of pure drug particles dispersed in an aqueous vehicle in which the diameter of the suspended particle is less than $1 \mu \mathrm{m}$ in size. The Nanosuspensions can also be lyophilized or spray dried and the nanoparticles of a Nanosuspension can also be incorporated in a solid matrix.

\section{Advantage [5-6]}

$\checkmark$ Only poorly soluble drug can be applied.

$\checkmark$ Rapid dissolution and tissue targeting can be achieved by IV Route. $\checkmark$ Reduced tissue irritation.

$\checkmark$ Improvement in biological performance due to higher dissolution rate and saturation solubility of drug.

$\checkmark \quad$ Long term physical stability in presence of stabilizer.

$\checkmark$ Higher bioavailability in case of ocular administration and inhalation drug delivery.

$\checkmark$ Nanosuspension can be incorporated into cream, gel, pellet, capsule and tablet.

\section{Disadvantages}

$\checkmark$ Physical stability, sedimentation and compaction can causes problems.

$\checkmark \quad$ It is bulky sufficient care must be taken during handling and transport.

$\checkmark$ Uniform and accurate dose cannot be achieved unless suspension are

$\checkmark \quad$ In a proper dose.

\section{Techniques for preparation of nanosuspensions [7]}

Technically preparations of nanosuspensions are simpler alternative than liposome's and other conventional colloidal drug carriers but reported to be more cost-effective. It is particularly for poorly soluble drugs and to yield a physically more stable product. For manufacturing nanosuspensions, there are two converse methods, "Top-down process technology" and "Bottom-up process technology".

The top-down process follows the disintegration approach from large particles, microparticles to Nanosized particles.

Examples are:

- High-pressure homogenization

- Nanoedge

- Nanopure

- Media milling (Nanocrystals). 
Bottom-up process is an assembly method forms nanoparticles from molecules.

Examples includes:

- Solvent-Antisolvent method

- Super critical fluid process

- Emulsification Solvent evaporation technique

- Lipid emulsion/Micro-emulsion template.

The principle techniques used in recent years for preparing nanosuspensions are:

\section{Wet milling [8-10]}

Nanosuspensions are produced by using high-shear media mills or pearl mills. The mill consists of a milling chamber, milling shaft and are circulation chamber. An aqueous suspension of the drug is then fed into the mill containing small grinding balls/pearls. As these balls rotate at a very high shear rate under controlled temperature, they fly through the grinding jar interior and impact against the sample on the opposite grinding jar wall. The combined forces of friction and impact produce a high degree of particle size reduction. The milling media or balls are made of ceramic-sintered aluminum oxide or zirconium oxide or highly cross-linked polystyrene resin with high abrasion resistance. Planetary ball mills (PM100 and PM200; Retsch GmbH and Co., KG, Haan, Germany) is one example of equipment that can be used to achieve a grind size below $0.1 \mu \mathrm{m}$. A nanosuspension of Zn-Insulin with a mean particle size of $150 \mathrm{~nm}$ was prepared using the wet milling technique. The major drawbacks of this technology include the erosion of balls/pearls that can leave residues as contaminants in the final product, degradation of the thermolabile drugs due to the heat generated during the process and presence of relatively high proportions of particles $\geq 5 \mu \mathrm{m}$.

\section{Homogenization dissocubes [11-14]}

Homogenization involves the forcing of the suspension under pressure through a valve having a narrow aperture. Dissocubes; was developed by Muller et al. in 1999. In this case, the suspension of the drug is made to pass through a small orifice that results in a reduction of the static pressure below the boiling pressure of water, which leads to boiling of water and formation of gas bubbles. When the suspension leaves the gap and normal air pressure is reached again, the bubbles implode and the surrounding part containing the drug particles rushes to the center and in the process colloids, causing a reduction in the particle size. Most of the cases require multiple passes or cycles through the homogenizer, which depends on the hardness of drug, the desired mean particle size and the required homogeneity. This principle is employed in the APV Gaulin Micron LAB 40Homogenizer (APV Homogenizer, Lóbeck, Germany) and the NS 1001L-Panda 2K high-pressure homogenizer (Nirosuavi. S. P. A., Parma, Italy). Scholer et al. prepared atovaquonenaa nosuspensions using this technique. An aqueous suspension of atovaquone was dispersed using an Ultra turrax T25, IKA-Werke Gmb H and Co. KG, Staufen, Germany and was further homogenized in a Gaulin Micron Lab 40 high-pressure homogenizer. After subjecting to pressures of $1.5 \times 107$ (two cycles), $5 \times 107$ (two cycles) and $1.5 \times 108$ (20 cycles) $\mathrm{Pa}$, ananosuspension of atovaquone with a mean diameter of $279 \pm 7 \mathrm{~nm}$ and mean poly dispersity index of $0.18 \pm 0.001$ was obtained. To produce ananosuspension with a higher concentration of solids, it is preferred to start homogenization with very fine drug particles, which can be accomplished by pre-milling. The major advantage of high-pressure homogenization over media milling is that it can be used for both diluted as well as concentrated suspensions and also allows aseptic production.

\section{Nanopure [15-18]}

Nanopure is suspensions homogenized in water-free media or water mixtures. In Dissocubes technology, the cavitation is the determining factor of the process. But, in contrast to water, oils and oily fatty acids have very low vapour pressure and a high boiling point. Hence, the drop of static pressure will not be sufficient enough to initiate cavitation. Patents covering disintegration of polymeric material by high-pressure homogenization mention that higher temperatures of about $80 \mathrm{C}$ promoted disintegration, which cannot be used for thermolabile compounds. Innanopure technology, the drug suspensions in thenon-aqueous media were homogenized at ${ }^{\circ} \mathrm{C}$ or even below the freezing point and hence are called "deep-freeze" homogenization. The results obtained were comparable to Dissocubes and hence can be used effectively for thermolabile substances at milder conditions.

\section{Nanoedge [14, 19]}

The basic principles of Nanoedge are the same at hat of precipitation and homogenization. A combination of these techniques results in smaller particle size and better stability in a shorter time. The major drawback of the precipitation technique, such as crystal growth and long-term stability, can be resolved using the Nanoedgetechnology. In this technique, the precipitated suspension is further homogenized, leading to a reduction in particle size and avoiding crystal growth. Precipitation is performed in water using water-miscible solvents such as methanol, ethanoland isopropanol. It is desirable to remove those solvents completely, although they can betolerated to a certain extent in the formulation. For an effective production of nanosuspensions using the Nanoedge technology, an evaporation step can be included to provide a solvent-free modified starting material followed by high-pressure homogenization.

\section{Nanojet-technology $[17,19]$}

This technique, called opposite stream or nanotechnology, uses a chamber where a stream of suspension is divided into two or more parts, which colloid with each other at high pressure.

The high shear force produced during the process results in particle size reduction. Equipment using this principle includes the M110L and M110 Smicrofluidizers (Microfluidics). Dearn prepared nanosuspensions of atovaquone using the microfluidization process. The major disadvantage of this technique is the high number of passes through the microfluidizer and that the product obtained contains a relatively larger fraction of microparticles.

\section{Emulsification-solvent evaporation technique [18, 19]}

This technique involves preparing a solution of drug followed by its emulsification in another liquid that is a non-solvent for the drug. Evaporation of the solvent leads to precipitation of the drug. Crystal growth and particle aggregation can be controlled by creating high shear forces using a high-speed stirrer.

\section{Hydrosol method [19]}

This is similar to the emulsification-solvent evaporation method. The only difference between the two methods is that the drug solvent is miscible with the drug anti-solvent. Higher shear force prevents crystal growth and Ostwald ripening and ensures that the precipitates remain smaller in size.

\section{Supercritical fluid method [20]}

Supercritical fluid technology can be used to produce nanoparticles from drug solutions. The various methods attempted are rapid expansion of supercritical solution process (RESS), supercritical antisolvent process and precipitation with compressed anti-solvent process (PCA). The RESS involves expansion of the drug solution in supercritical fluid through anozzle, which leads to loss of solvent power ofthe supercritical fluid resulting in precipitation ofthe drug as fine particles. Young et al. Prepared cyclosporine nanoparticles in the size range of 400-700 $\mathrm{nm}$ using this process. In the PCA method, the drug solution is atomized into a chamber containing compressed $\mathrm{CO} 2$. As the solvent is removed, the solution gets supersaturated and thus precipitates as fine crystals. The supercritical anti-solvent processuses a supercritical fluid in which a drug is poorly soluble and a solvent for the drug that is also miscible with the supercritical fluid. The drug solution is injected into the supercritical fluid and the solvent gets extracted by the supercritical fluid and the drug solution gets supersaturated. The drug is then precipitated as fine crystals. Nanoparticles of griseofulvin, a drug with poor solubility, were 
prepared by Chattopadhyay et al. using this method. The disadvantages of the above methods are use of hazardous solvents and use of high proportions of surfactants and stabilizers as compared with other techniques, particle nucleation overgrowth due to transient high supersaturation, which may also result in the development of an amorphous form or another undesired polymorph.

\section{Evaluation parameters [17-21]}

The Nanosuspension was evaluated for various parameters:

$$
\begin{array}{ll}
\checkmark & \text { Content uniformity } \\
\checkmark & \text { pH } \\
\checkmark & \text { Particles size and shape } \\
\checkmark & \text { In vitro drug release studies }
\end{array}
$$

\section{Drug content uniformity}

$10 \mathrm{ml}$ of each formulation was taken and dissolved in $10 \mathrm{ml}$ isotonic solution and kept overnight. $10 \mathrm{mg}$ (similar as in formulation) of drug was taken and dilution was made to $10 \mu \mathrm{g} / \mathrm{ml}$. The dilutions were filtered and analyzed using UV for their content uniformity. The absorbance of the formulations were read using one $\mathrm{cm}$ cell in a UV-Vis spectrophotometer. The instrument was set at $245 \mathrm{~nm}$. The drug content in each formulation was calculated based on the absorbance values of known standard solutions.

\section{pH}

The pH values were measured at 25 ac using a pH digital meter at $20 \pm 1$ ? C. The formulation was brought in contact with the electrode of $\mathrm{pH}$ meter and equilibrated for $1 \mathrm{~min}$. This method was done in triplicate and mean was calculated along with standard deviation.

\section{Particle size and shape}

Particle size and shape of the formulated Nanosuspension was determined by using Scanning Electron Microscopy.

\section{In vitro drug release}

In vitro drug release studies were performed in a dissolution apparatus using paddle method at rotation speed of $50 \mathrm{rpm}$. The volume and temperature of the dissolution medium were $900 \mathrm{ml}$ and $37.0 \pm 0.2{ }^{\circ} \mathrm{C}$, respectively. Samples were withdrawn at fixed times and were filtered and assayed through ultraviolet absorbance determination at $245 \mathrm{~nm}$ using a Shimadzu UV-Visible spectrophotometer.

\section{CONCLUSION}

Nanosuspension solved poor bioavailability problem of hydrophobic drugs and drugs which are poorly soluble in aqueous and organic solutions. Large scale production of nanosuspension can be employed by media milling and high pressure homogenization technique. This technology is gaining significance as the number of molecules with solubility and bioavailability related problems are increasing day by day. Thus, nanotechnology can play a vital role in drug discovery programs to increase aqueous solubility as well as bioavailability of poorly soluble drugs.

\section{AUTHORS CONTRIBUTIONS}

All the author have contributed equally

\section{CONFLICT OF INTERESTS}

Declare none

\section{REFERENCES}

1. N Arunkumar, M Deecaraman, C Rani. Nanosuspension technology and its applicationsin drug delivery. Asian J Pharma 2009;13:168-73.

2. R Yadollahi, K Vasilev, S Simovic. Nanosuspension technologies for delivery of poorly soluble drugs. Hindawi Publishing Corporation J Nanomaterials 2005;7:1-9.

3. D Bhowmik, G Harish, S Duraivel, BP Kumar, V Raghuvanshi, KPS Kumar. Nanosuspension-a novel approaches in drug delivery system. Pharma Innovation J 2013;12:50-63.

4. RL Shid, SN Dhole, N Kulkarni, SL Shid. Nanosuspension: a review. Int J Pharm Sci Rev Res 2013;22:98-106.

5. KAK Verma. Nanosuspensions: advantages and disadvantages. Indian J Novel Drug Delivery 2012;4:179-88.

6. S Katteboinaa. Drug nanocrystals: a novel formulation approach for poorly soluble drugs. Int J Pharmtech Res 2009;1:682-94.

7. AP Patel. A review on drug nanocrystal a carrier free drug delivery. Int J Res Ayurveda Pharm 2011;2:448-58.

8. BP Patel. A review on techniques which are useful for solubility enhancement of poorly water-soluble drugs. Int J For Res Management Pharm 2012;1:56-70.

9. SA Patil, BR Rane, SR Bakliwal, SP Pawar. Nanosuspension: at a glance. Int J Pharm Sci 2011;3:947-60.

10. S Varun, R Kapoor, K Preet. Taste masking and formulation of ofloxacin rapid disintegrating tablets and oral suspension. Indian J Pharm Educ Res 2009;43:150-5.

11. RH Müller, C Jacobs, 0 Kayer. Nanosuspensions for the formulation of poorly soluble drugs. In: F Nielloud, G MartiMestres. (ed). Pharmaceutical emulsion and suspension. New York, Marcel Dekker; 2000. p. 383-407.

12. RH Muller, K Peters. Nanosuspensions for the formulation of poorly soluble drug I: preparation by size reduction technique. Int J Pharm Res 1998;160:229-37.

13. T Shah, D Patel, J Hirani, AF Amin. Nanosuspensions as a drug deliverysystem: a comprehensive review. Int J Pharma 2007;7:42-53.

14. GGeetha U Poojitha, AA Khan. Various techniques for preparation of nanosuspension-a review. Int J Pharma Res Rev 2014;3:30-7.

15. J Chingunpituk. Nanosuspension technology for drug delivery. Walailak J Sci Technol 2007;4:139-53.

16. N Arunkumar, M Deecaraman, C Rani. Nanosuspension technology and its applications in drug delivery. Asian J Pharm 2009;9:168-73.

17. M Mudgil, N Gupta, M Nagpal, P Pawar. Nanotechnology: a new approach for ocular drug delivery system. Int J Pharm Pharm Sci 2012;4:105-12.

18. GV Yadav. Nanosuspension: a promising drug delivery system. Pharmacophore 2012;3:217-43.

19. K Ezeddin. Nanodispersions platform for solubility improvement. Int J Res Pharm Biomed Sci 2013;4:636-43.

20. D Prasanta. Nanotechnology for the delivery of poorly water soluble drugs. Global J Pharma Res 2012;1:225-50.

21. C Toshi. A review on nanosuspensions promising drug delivery strategy. Curr Pharma Res 2012;3:764-76. 